\title{
Practice based research in dentistry: an alternative to deal with clinical questions
}

\author{
Flávio Fernando DEMARCO (a) \\ Marcos Britto CORREA(a) iD \\ Maximiliano Sérgio CENCI(a) iD \\ Frederick James Trevor BURKE(b) \\ Niek Johannes Maria OPDAM(c) \\ Andre Luis FARIA-E-SILVA(d) \\ (a) Universidade Federal de Pelotas - UFPel, \\ School of Dentistry, Graduate Program in \\ Dentistry, Pelotas, RS, Brazil. \\ (b) University of Birmingham, School of \\ Dentistry, Birmingham, UK. \\ (c) Radboud Institute for Health Sciences, \\ Department Radboud University Medical \\ Center, of Dentistry, Nijmegen, The \\ Netherlands. \\ (d) Universidade Federal de Sergipe - UFS, \\ Graduate Program in Dentistry, Aracaju, \\ SE, Brazil.
}

Declaration of Interests: The authors certify that they have no commercial or associative interest that represents a conflict of interest in connection with the manuscript.

Corresponding Author:

Flávio Fernando Demarco

flavio.demarco@ufpel.edu.br

https://doi.org/10.1590/1807-3107bor-2020.vol34.0071

Submitted: Sep 21, 2019

Accepted for publication: Nov 1, 2019

Last revision: June 8, 2020

\begin{abstract}
Clinical interventions in dental practice should be determined based on the best scientific evidence available. Well-designed randomized clinical trials (RCTs) provide important evidence supporting the efficacy of interventions and are usually considered as the best primary evidence. However, the strict criteria adopted by most RCTs reduce their external validity since some findings from these studies might not work under usual conditions. On the other hand, practice-based research (PBR) studies have been designed to better define the effectiveness of clinical interventions under settings closer to "real-world" conditions. Therefore, this review aimed to describe different PBR designs discussing some advantages and limitations of such studies. The stimulus to organization PBR networks is discussed since the studies performed by these networks involve large number of clinicians and important conclusions can be drawn. Designs of observational studies including surveys and cohort studies based on practice are presented. Survey methods are important to know the behavior of practitioners regarding diagnostic and decision of treatment. Cohorts allow assessing different cofounders contributing to some outcome since large sample sizes and long followup periods can be observed in some of these studies. Pragmatic trials designed to take place in real-world clinical practice settings are also discussed as a useful design to assess the effectiveness of clinical interventions. In conclusion, this review sought to present PBR studies as alternative designs to answer clinical questions, but not replacing randomized clinical trials.
\end{abstract}

Keywords: Clinical Trial; Evidence-Based Practice; Pragmatic Clinical Trial; Surveys and Questionnaires.

\section{Introduction}

Oral health constitutes part of general health and problems affecting oral cavity can negatively impair physical, psychological, emotional, and social aspects of the individuals' life. ${ }^{1}$ Despite the decline of oral health disease worldwide, their prevalence is still high, with untreated caries in the permanent dentition affecting about $35 \%$ of the global population. ${ }^{2}$ The cost to treat dental disease has a high individual and public system burden, being estimated that almost $5 \%$ of global health costs would be needed to treat such diseases. ${ }^{3}$ 
The approach to select interventions or strategies for treatment of oral conditions should be based on the best scientific evidence available. Systematic reviews/meta-analysis of randomized clinical trials are overall regarded as the best evidence source to determine the true response for a determined treatment, while randomized clinical trials are considered the best primary study design. ${ }^{4}$

However, there are some limitations to carry out RCTs in dentistry, including ethical aspects, the high cost and the need for long periods of follow-up to observe relevant outcomes. Usually, the criteria for inclusion and exclusion of patients, the standardization of interventions and outcomes in the RCTs are so restrict that the results of the trial would have high internal validity (efficacy), but limited implication to the external population (efficiency). ${ }^{5}$ Additionally, there is a huge gap between obtaining the scientific evidence and its translation to practice. ${ }^{6}$

In order to provide studies that mimic the "real" world of the dentistry field, practice-based research studies have been used as a different methodological approach. Practice-based studies are developed inside the clinical practice, by clinicians to try to answer questions raised from the patients' daily care so that the results could be compared in a more reliable way to the general population. The difference between patients are statistically controlled and the confusion factors that could affect treatment outcome are included. ${ }^{7}$ In PBR studies usually more than one hypothesis could be tested and new hypothesis could be generated during the study. Moreover, the involvement of patients and practioners in the research regarding data collection can help with the translations of the findings of practice-based studies to their direct application. ${ }^{8}$

Practice-based research studies may have a wide range of designs, being used for several different purposes, such as asking dentists about their choices in clinical practice, following dental treatments overtime in a large number of patients or using pragmatic design to test different research questions with higher inference power.

The aim of this review paper is to present the different application of Practice-based research studies, discussing possibilities from observational to experimental designs, pointing out the advantages and limitations of each methodological approach and providing an overview in relation to the state of art for such studies.

\section{Defining practice-based research initiatives and networks}

As a general definition, any piece of research that takes the nature of practice as its central focus is called "practice-based" research. This type of research is acknowledged in all fields and is not restricted to biomedical sciences. In biomedical sciences, a simple search in Pubmed shows that the first hits for the terminology "practice-based research" are from the second half of the last century, and thousands of papers are indexed under this specific term. In dental sciences, the first hits in Pubmed are from the 1970's, and the amount of original publications as largely increased in the 2000's.

Practice-based research has several advantages when compared to research conducted more "artificial" environments, such as in dental schools and other academic settings. It uses real-world patients, and realworld dental health care workers, who are subjected to all the pressures of running a busy, but financially viable practice. ${ }^{9}$ It also usually deals (or should deal) with clinically relevant problems for patients and clinicians, while several academic clinical studies are reporting on surrogate endpoints.

Practice-based research networks (PBRN) are large organizations of practitioners that are recruited and who agree on taking part on research projects and on data collection. Dental PBRNs started to be organized over the last 15 years, in order to generate evidence-based knowledge with good external validity and to facilitate the adoption of the research findings by involving practitioners in the research process. This process was mostly stimulated by the United States National Institute of Dental and Craniofacial Research (NIDCR) that began funding PBRNs in 2005. In 2012, NIDCR funded a single, integrated national network, the National Dental Practice-Based Research Network. ${ }^{8,10}$ This Network has enrolled more than 2700 participants from several regions in US, and has published a considerable amount of outcomes. 


\section{Observational studies}

Observational designs are characterized by the absence of any intervention by researchers. There is a high variability of methodological approaches that can be used in a practice-based perspective, from simple cross-sectional surveys to cohorts with long-term follow-ups. Each of these designs is capable of answering specific questions, depending on the research question.

\section{Surveys}

Cross-sectional surveys are the simplest method to apply in a practice-based perspective. In general, surveys are useful to investigate the opinion or the attitudes of professionals regarding a topic of interest. For example, the study of Nascimento et al..$^{11}$ investigated which material is the first choice of general dentists from a Brazilian southern city to restore posterior teeth. Two points are crucial in this type of study to ensure representativeness of the interest population: sampling and delivery of the instrument.

Regarding sample selection, the intention is that the results of the sample could be generalized for a specific population. Using the study of Nascimento et al. ${ }^{11}$ as an example, since the intention was to generalize the results from general practitioners from the city of Pelotas, to select the sample in an unbiased way, a list of practitioners registered in the city was obtained. In general, representative samples are easier to obtain when small populations are being investigated. On the other hand, the external validity will be higher in studies with a wider range of target population, for example, general dentists from a whole country.

Aspects that should be considered in questionnaire application include when, where and how the instrument will be administered. The response rate will depend mainly on these variables. Delivery of questionnaires personally at dentists' workplace ensures higher response rates but needs greater human resources. Actually, there are different applications available to apply questionnaires online. This approach can maximize the coverage of delivery, but response rates are much lower compared to personal delivery. For example, a recent study intended to investigate the use of scientific evidence among dentists working in Brazil. ${ }^{12}$ Invitations to answer an electronic questionnaire were sent by email by the regional councils of dentistry of each Brazilian state. The response rate was very low, as it usually happens with the online approach, being estimated a participation of $0.3 \%$ of all dentists registered in Brazil. The time to fill out the questionnaire is also an important aspect. Questionnaires should be designed as short as possible to reduce time of application that will result in higher response rates.

A type of survey that is of special interest in practice-based research is decision-making studies. In these studies, clinicians are asked to give their opinion about treatment/diagnosis decision while confronted with specific clinical situations presented in a questionnaire. This methodological approach is useful to understand the management of clinical situations in real world, which in general is different from the research perspective. Signori et al. ${ }^{13}$, investigated the decision-making of general dental practitioners (GDPs) regarding bitewing radiographs of proximal restorations, comparing with the opinion of experts in cariology and restorative dentistry, showing that GDPs tend to have a less conservative approach regarding the decision to intervene or not concerning the reassessment of restorations.

Decision-making studies are also a good alternative to explore one of the less investigated topics in dentistry, the role that professionals have on treatments. In this way, it is possible to combine methods, using decision-making surveys varying characteristics of the case, for example, patient characteristics. In this way, a recent study aimed to investigate if skin color of patient affects the decision of dentists. ${ }^{14}$ Two questionnaires were produced for the same clinical case, but the images were digitally manipulated to obtain a patient with a black and a white skin color. The skin color was randomly assigned previously to the questionnaire application. The results showed that patient skin color influenced the dentist's choice of treatment. In general, black patients receive referrals for cheaper, simpler procedures suggesting that professionals may contribute unconsciously to the propagation and replication of racial discrimination. This type of methodological approach can be used to investigate how other patient-related aspects can affect the decision of clinicians. 


\section{Cohort Studies}

\section{Population-based cohorts}

The longitudinal investigation of clinical outcomes in population-based samples is a good alternative to explore in practice-based research how individual-related variables affect dental treatments. In birth cohorts, all individuals born in the same year and at the same place are followed-up to investigate the effect of exposures on future outcomes. The large sample, the long period of follow-up and the involvement of a team of researchers make cohort studies expensive to maintain on long term. Normally these studies are multidisciplinary, with several research questions. This characteristic provides access to a high number of exposure variables, with high variability, which is difficult to obtain in clinical prospective or retrospective studies. A good example of this design are the studies on posterior restorations in Pelotas 1982 birth cohort. ${ }^{15,16}$ These studies showed the effect that individual-related variables, such as the socioeconomic trajectories and the caries risk from 15 to 31 years of age, experienced in the life course has on quality of restorations.

Despite the wide range of individuals' data available, population-based designs present as their main limitation the difficulty of controlling clinical data. Generally, cohort studies assess individuals but fail to present detailed variables on dental treatments because dentists are not investigated. In countries where patient electronic files are available this problem can be solved. As alternative, the use of non-reversible outcomes is a possibility. For example, Chisini et al. ${ }^{17}$ investigated factors associated with replacement of amalgam restorations by composites from 24 to 31 years of age in 1982 Pelotas birth cohort. Even without contact with clinical files, the replacement of materials is evident in an epidemiological assessment, which would be impossible in case of replacement of an old composite restoration by a new one. Other possibility is to consider as treatment outcome the tooth loss. In this case, it is possible to calculate survival of teeth after clinical interventions.

\section{Retrospective longitudinal studies}

In dental literature, a large number of studies have been classified as "retrospective longitudinal studies". As an example, for restorative dentistry, a crescent number of primary studies and reviews were published covering this topic. ${ }^{18,19,20}$ These retrospective studies include the report of a series of cases of single or multiple dental practices, in which patient file record files are surveyed to look at the outcome of dental treatments. This type of design allows assessing several years after the dental intervention. In some retrospective studies, independent evaluators go to the dental practice and make an independent evaluation of the treatments in loco, using visual inspection and other tools to collect data. ${ }^{21}$ While in other retrospective studies, only data from files are used to do the report. ${ }^{22}$ Considering that these data are retrieved from the dental practices, these studies are PBR studies in their nature. These retrospective studies have as their most significant advantage the possibility of longer follow-up times compared to, for instance, RCTs, which in dentistry usually have a limited duration. Another advantage of these studies, due to their PBR nature, is that they typically allow data collection for a broader source of patient and dental service profiles. This allows, for instance, the investigation of patient risk factors and variations among clinicians in general practice.

However, some limitations of these nonrandomized studies should be disclosed..$^{20}$ These studies are under risk of selection bias, as dentists invariably choose the interventions according to their judgment criteria. Selection bias is especially sensitive in cases where the retrospective study aims to compare treatment options. In these comparative studies, the clinician is probably indicating different types of interventions according to distinct clinical conditions. Also, reporting bias and the reliability of clinical records should always be taken into account in these studies. These records where not intended for data collection and research purposes at the beginning, and therefore missing or conflicting data are always a possibility in retrospective studies. Some imprecision or contamination of data should also be taken in account, because patients could, for instance, visited other dental practice and undertaken other treatments, and this may not be reported in the dental records screened for research. 


\section{Experimental studies - from explanatory RCT's to pragmatic trials}

Practice-based study settings, in especial networks, are a very interesting environment to develop experimental studies with "real life" appeal. Whereas seldom explored, this possibility could help dental research to fill up important gaps in the knowledge, and especially, to help in the achievement of the best evidence for clinical practice. In a general sense, the development of prospective studies in the context of PBR has similar challenges and advantages compared to the traditional academic or hospital environment. But some extra challenges should be taken into account, especially concerning the lack of strict control by the researcher on what is going on in the trial (i.e., are the operators following the protocols? Are they always respecting the randomization?). In this context, a traditional explanatory trial would be really difficult to implement in PBR, given room for the proposal of more pragmatic trials for the achievement of clinically contextualized finds.

Despite their importance in determining the efficacy of any treatment, some aspects of RCTs (explanatory studies), such as usual strict inclusion criteria and data collection, reduce the external validity of outcomes compromising the application of the findings in the clinical routine ("real-world"). 5,23 In fact, it is commonly observed that the findings of explanatory trials are less effective in practice than they were in controlled settings since several factors (e.g. pre-conditions affecting the outcomes) closely related to "real world" are excluded from those studies. ${ }^{24,25}$ On the other hand, pragmatic trials are designed to take place in real-world clinical practice settings, where typical patients receive the interventions by clinicians who sometimes did not have any research background. ${ }^{26}$ In order to achieve this purpose, pragmatic trials must enroll participants from heterogeneous practice settings, including a broad range of demographic features, to assess several significant outcomes from comparisons involving clinically relevant alternative interventions. ${ }^{27} \mathrm{~A}$ recommended approach is to run the pragmatic trials in settings where patients already receive their usual clinical care maintaining several aspects inherent to their clinical routine. ${ }^{28}$
These aspects maximize the external validity of the trial allowing to generalize the findings to many real-world settings, aiding to explain the pervasive and persistent unexplained variability observed in clinical practice.

The term "pragmatic" was coined to describe studies seeking to help the decision-makers in choosing the best option of healthcare instead of elucidating the efficacy of a treatment. ${ }^{26}$ The differences between explanatory and pragmatic trials can be summarized based on the questions that these study methods aim to answer: "Can this intervention work under ideal conditions?" or "Does this intervention work under usual conditions?", respectively. ${ }^{26}$ Thus, explanatory trials seek to understand the biological or mechanistic aspects of an intervention, whereas pragmatic trials are designed to inform the decision-makers (patients, clinicians, administrators, and policymakers) of which intervention is preferable to solve some health issue. Unlike most RCTs comparing alternative interventions to placebo or absence of intervention, pragmatic trials are designed to compare different interventions already used in the clinical practices facing the choices available for patients and their physicians. ${ }^{29}$ Despite observational and other nonexperimental studies also allowing to assess the effectiveness of clinical interventions, these methods usually fail to provide robust evidence to support alternative clinical intervention since important cofounders are not controlled.

Furthermore, in order to recruit participants from a variety of practice settings, pragmatic studies must also measure a broad range of relevant clinical outcomes considering their importance to define changes on clinical practice. ${ }^{27}$ Therefore, several patient-based outcomes such as quality of life and satisfaction, and analysis of cost-effectiveness are included to support the definitions of best intervention to be implemented. Another important point is that the periods of follow-up in pragmatic studies are usually longer than those observed in most RCTs. ${ }^{27}$ Extending the follow-up improves the ability to determine the real effect of a clinical intervention, whereas some outcomes observed after short period of follow-up can be related to a deficient implementation of intervention. ${ }^{30}$ 
It is important to emphasize that most trials are not purely pragmatic or explanatory, and a randomized trial can take over several aspects of pragmatic design. ${ }^{29}$ Thus, the definition of a trial as pragmatic or explanatory involves several dimensions rather than a dichotomy classification. A useful tool developed to assess the pragmatic aspects of a trial was the pragmatic-explanatory continuum indicator summary (PRECIS). ${ }^{31}$ This tool consisted of ten domains aiming to determine the extent to which a trial is pragmatic or explanatory. The revised version (PRECIS-2) contains the following nine domains: a) Similarity of eligible participants with those observed in the usual care, b) the effort done to recruit participants over and above those in usual care settings, c) differences between the settings of the trial from the usual care setting, d) differences between the organization delivering the intervention and those available in usual care, e) flexibility of intervention delivered, f) flexibility of how participants are monitored and encouraged to adhere to the intervention, g) intensity and period of follow-up, h) direct relevance of primary outcome, and i) data inclusion in the analysis. ${ }^{32}$ Each domain is scored on a 5 -point Likert continuum (from 1 = very explanatory "ideal conditions" to $5=$ very pragmatic "usual care conditions") allowing to assess the pragmatic feature of study design.

Even tough pragmatic trials are important study designs to support the effectiveness of clinical practice, clinical trials using a pragmatic approach seem to be scarce in dentistry. We performed a systematic search using the electronic database MEDLINE via PubMed on July 9, 2019 to estimate the number of pragmatic trials published in dentistry. Initially, the search was done to identify the clinical trials in dentistry published in journals indexed to this database and we obtained a result of 14,139 articles. A second search done for pragmatic trials (not only for dentistry) in which 215 articles were identified. Only a single article ${ }^{33}$ was identified when the two strategies were combined, demonstrating the lack of pragmatic clinical trials in dentistry (Table). This identified study ${ }^{33}$ performed in three primary schools aimed to evaluate whether the frequency of chewing sugar-free gum aids on reducing the extension of white-spot lesions measured by quantitative lightinduced fluorescence (surrogate outcome). However, assessing the pragmatic characteristic of this trial using the PRECIS-2 diagram tool resulted in total score of 19 points in a scale ranging from 9 (very explanatory) to 45 (very pragmatic) (Figure). Therefore, the only pragmatic study identified presents several features of explanatory trials rather than pragmatic.

There are some initiatives proposing pragmatic trials in the context of PBRN. One good example is the ongoing trial in the Dutch PBRN, ${ }^{34}$ which aims to investigate the effectiveness of antimicrobial containing dental adhesives for the prevention of secondary caries. For this study, a cohort of dental practices with motivation to participate and that are acknowledged for keeping very good dental records were invited to take part in the study. The same dentist (operators) will be using one adhesive system with antimicrobial properties for a certain period of time, and later switch for a control adhesive system, in a sort of cross-over design. The primary outcome (presence of new secondary caries lesions) is going to be collected after 5 year of follow-up.

Table. Search strategy used to identify pragmatic clinical trials and the results from searching in Pubmed database.

\begin{tabular}{|c|c|c|c|c|}
\hline Search & Add to builder & Query & Items found & Time \\
\hline \#3 & Add & Search (\# 1 AND \#2) & 1 & $09: 20: 16$ \\
\hline$\# 2$ & Add & $\begin{array}{c}\text { Search (Pragmatic Clinical Trials as Topic [MeSH Terms]) AND (Naturalistic Randomized } \\
\text { Clinical Trial [Title/Abstract] OR Practical Clinical Trials [Title/Abstract] OR Clinical } \\
\text { Trials, Practical [Title/Abstract] OR Trials, Practical Clinical [Title/Abstract] OR Pragmatic } \\
\text { Trials [Title/Abstract] OR Trials, Pragmatic [Title/Abstract] OR Pragmatic Clinical Trials } \\
\text { [Title/Abstract] OR Clinical Trials, Pragmatic [Title/Abstract] OR Trials, Pragmatic Clinical } \\
\text { [Title/Abstract] OR Real World Clinical Trials [Title/Abstract])) }\end{array}$ & 215 & $09: 19: 38$ \\
\hline 31 & Add & $\begin{array}{c}\text { Search ((dentistry [MeSH Terms])) AND (((randomized controlled trial [Publication } \\
\text { Type] OR (randomized [Title/Abstract] AND controlled [Title/Abstrct] AND trial [Title/ } \\
\text { Abstract])))) }\end{array}$ & 14139 & $09: 18: 37$ \\
\hline
\end{tabular}




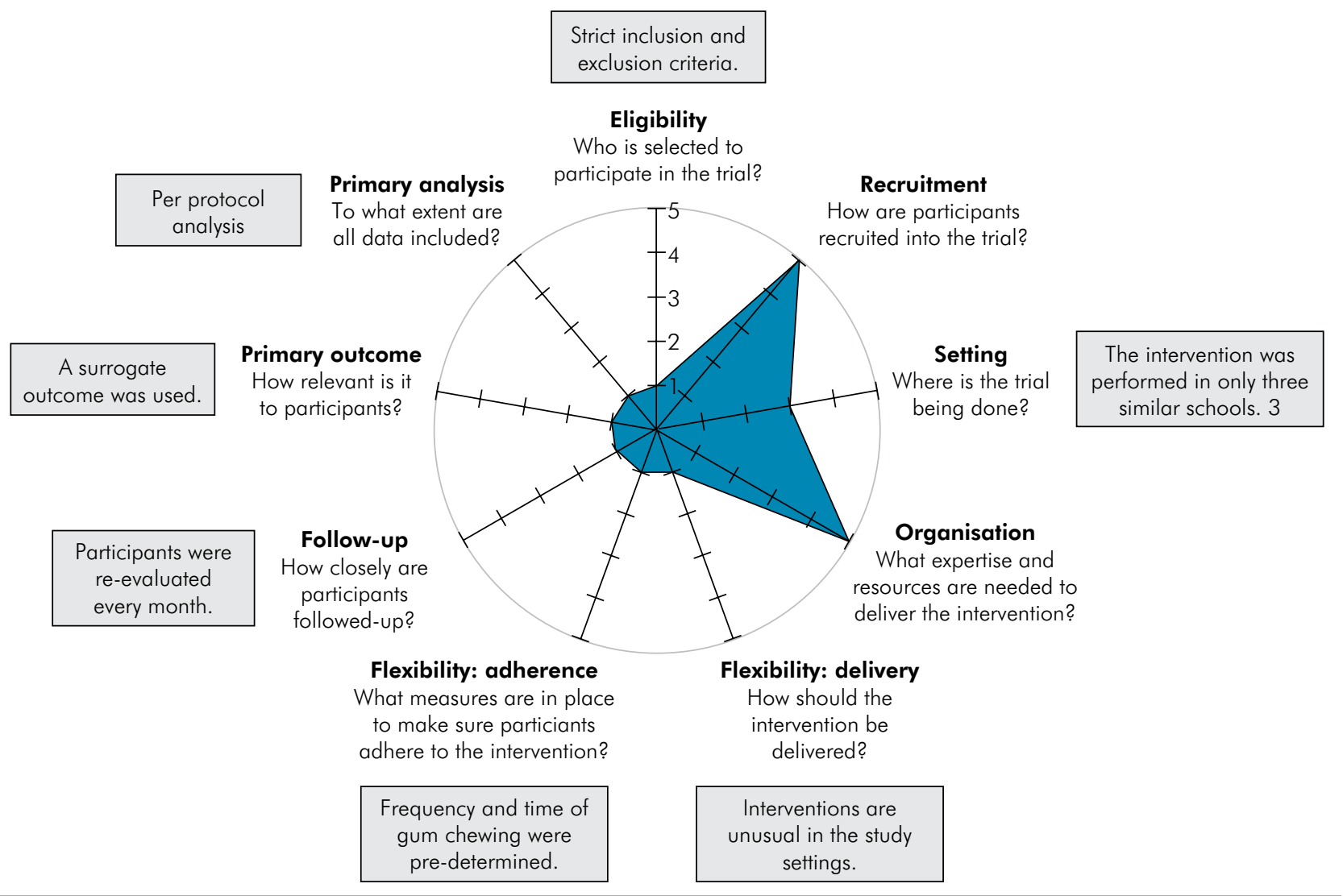

Figure. Pragmatism assessment of the identified trial using the pragmatic-explanatory continuum indicator summary (PRECIS2) diagram tool.

\section{Discussion}

Practice-based research can only be successful in environments where the participants (practitioners) are motivated to take part in the research. Therefore, if the participants have some compensation, such as financial reimbursement, or credits for continuum education, they are probably going to be more prone to be engaged and deliver data with higher precision. In some situations where there is funding for an extensive network, as the National Dental PBRN Collaborative Group in US, the survival of the network and the outcomes are more predictable..$^{35}$ However, in other countries, the funding limitation prevents more significant initiatives. Even though practice-based studies have been published from several countries such as Scandinavia, UK, Netherlands, Germany, Brazil, US, Australia.

One of the main issues for practice-based research outcomes is the representativity of the population of dentists or patients in a national system. Usually, as only the more motivated dentists will take part in these PBR initiatives, it would be virtually impossible to have a general picture of a country, unless data are acquired directly from electronic record files at a national level. While these national electronic files are more popular these days, they are currently developed for reimbursement purposes and mostly not for research data collection. Moreover, most of the electronic record systems are not currently covering all dimensions of dental practices in a country. In this context, it is possible to assume that even if PBR studies are considered more connected with "real world dentistry," and even when if PBR studies are planned to have higher external validity, representativeness will still be an issue of concern.

Another challenge for implementation of PBRNs is to close the existent gap between professional and academic worlds. In most of cases, research problems are defined by researchers, which not 
necessarily represent real clinical problems faced by practitioners. Makansi et al. ${ }^{36}$ reported that researchers and practitioners present different motivations to join on PBRNs. While practitioners viewed the network as an alternative to reduce professional isolation, researchers interpreted as a way to produce more research. In addition, practitioners found research topics not very useful, despite researchers' belief that studies were interesting and relevant for dental practice. ${ }^{36}$

In this paper, we covered a broad spectrum of possibilities for using PBR in different study designs. However, for each study design, the conventional methodological aspects should be taken into account to produce the best conduction and reporting of these studies, and all the limitations should be disclosed to avoid spin in science and misinterpretation.

The future of PBR studies is auspicious, considering the increasing availability of data from large national electronic record systems. From this perspective, information can be more accessible and more transparently and reproducibly retrieved. Also, the digital era allows already the overcoming of the old fashion intra-oral inspection by a single or a panel of evaluators, allowing the replacement for indirect methods such as scanning, intraoral photography or other image systems that will enable to make independent evaluations on collected data. Altogether, these tools will bring PBR studies to a new level, with great possibilities to unbox the "real world dentistry" and bring more precise data to improve dental science and dental practice.

\section{Conclusions}

Considering the discussion throughout the manuscript, the PBR studies are an excellent methodological tool to develop studies mimicking the real-world dentistry, with a great external validity and therefore their findings could be more easily translated to the dental practice. PBR studies could be used for a series of purposes, including surveys about dentists' preferences, long term retrospective/prospective evaluation of treatments, or pragmatic designs testing different hypothesis. Also, PBR approaches could be used in different areas of dentistry. However, the PBR studies should not be considered a replacement for RCTs or any kind of traditional study design, instead they should be treated as a complementary weapon in the arsenal of methodologies to investigate treatments or techniques options.

\section{References}

1. Peres MA, Macpherson LM, Weyant RJ, Daly B, Venturelli R, Mathur MR, et al. Oral diseases: a global public health challenge. Lancet. 2019 Jul;394(10194):249-60. https://doi.org/10.1016/S0140-6736(19)31146-8

2. Marcenes W, Kassebaum NJ, Bernabé E, Flaxman A, Naghavi M, Lopez A, et al. Global burden of oral conditions in 1990-2010: a systematic analysis. J Dent Res. 2013 Jul;92(7):592-7. https://doi.org/10.1177/0022034513490168

3. Listl S, Galloway J, Mossey PA, Marcenes W. Global economic impact of dental diseases. J Dent Res. 2015 Oct;94(10):1355-61. https://doi.org/10.1177/0022034515602879

4. Murad MH, Asi N, Alsawas M, Alahdab F. New evidence pyramid. Evid Based Med. 2016 Aug;21(4):125-7. https://doi.org/10.1136/ebmed-2016-110401

5. Schwendicke F, Opdam N. Clinical studies in restorative dentistry: design, conduct, analysis. Dent Mater. 2018 Jan;34(1):29-39. https://doi.org/10.1016/i.dental.2017.09.009

6. Contopoulos-loannidis DG, Alexiou GA, Gouvias TC, loannidis JP. Medicine. Life cycle of translational research for medical interventions. Science. 2008 Sep;321(5894):1298-9. https://doi.org/10.1126/science.1160622

7. Horn SD, Gassaway J. Practice-based evidence study design for comparative effectiveness research. Med Care. 2007 Oct;45(10 Supl 2):S50-7. https://doi.org/10.1097/MLR.0b013e318070c07b

8. Gordan VV, Makhija SK, Rindal DB, Meyerowitz C, Fellows JL, Ziegenfuss JY, et al. Leadership in practice-based research: the National Dental PBRN. J Dent. 2019 Aug;87:24-7. https://doi.org/10.1016/i.jdent.2019.05.009

9. Burke FJ, Stewardson D. Practice-based research. J Am Dent Assoc. 2005 Oct;136(10):1362-4. https://doi.org/10.14219/jada.archive.2005.0039 
10. Gilbert GH, Williams OD, Korelitz JJ, Fellows JL, Gordan VV, Makhija SK, et al. Purpose, structure, and function of the United States National Dental Practice-Based Research Network. J Dent. 2013 Nov;41(11):1051-9. https://doi.org/10.1016/i.jdent.2013.04.002

11. Nascimento GG, Correa MB, Opdam N, Demarco FF. Do clinical experience time and postgraduate training influence the choice of materials for posterior restorations? Results of a survey with Brazilian general dentists. Braz Dent J. 2013 Nov-Dec;24(6):642-6. https://doi.org/10.1590/0103-6440201302361

12. Gonçalves AP, Correa MB, Nahsan FP, Soares CJ, Moraes RR. Use of scientific evidence by dentists in Brazil: room for improving the evidence-based practice. PLoS One. 2018 Sep;13(9):e0203284. https://doi.org/10.1371/journal.pone.0203284

13. Signori C, Laske M, Mendes FM, Huysmans MD, Cenci MS, Opdam NJ. Decision-making of general practitioners on interventions at restorations based on bitewing radiographs. J Dent. 2018 Sep;76:109-16. https://doi.org/10.1016/i.jdent.2018.07.003

14. Chisini LA, Noronha TG, Ramos EC, Santos-Junior RB, Sampaio KH, Faria-e-Silva AL, et al. Does the skin color of patients influence the treatment decision-making of dentists? A randomized questionnaire-based study. Clin Oral Investig. 2019 Mar;23(3):1023-30. https://doi.org/10.1007/s00784-018-2526-7

15. Collares K, Opdam NJ, Peres KG, Peres MA, Horta BL, Demarco FF, et al. Higher experience of caries and lower income trajectory influence the quality of restorations: A multilevel analysis in a birth cohort. J Dent. 2018 Jan;68:79-84. https://doi.org/10.1016/i.jdent.2017.11.009

16. Correa MB, Peres MA, Peres KG, Horta BL, Barros AJ, Demarco FF. Do socioeconomic determinants affect the quality of posterior dental restorations? A multilevel approach. J Dent. 2013 Nov;41(11):960-7. https://doi.org/10.1016/i.jdent.2013.02.010

17. Chisini LA, Collares K, Bastos JL, Peres KG, Peres MA, Horta BL, et al. Skin color affect the replacement of amalgam for composite in posterior restorations: a birth-cohort study. Braz Oral Res. 2019 Jul;33:e54. https://doi.org/10.1590/1807-3107bor-2019.vol33.0054

18. Opdam NJ, Sande FH, Bronkhorst E, Cenci MS, Bottenberg P, Pallesen U, et al. Longevity of posterior composite restorations: a systematic review and meta-analysis. J Dent Res. 2014 Oct;93(10):943-9. https://doi.org/10.1177/0022034514544217

19. Demarco FF, Collares K, Coelho-de-Souza FH, Correa MB, Cenci MS, Moraes RR, et al. Anterior composite restorations: A systematic review on long-term survival and reasons for failure. Dent Mater. 2015 Oct;31(10):1214-24. https://doi.org/10.1016/i.dental.2015.07.005

20. Opdam NJ, Collares K, Hickel R, Bayne SC, Loomans BA, Cenci MS, et al. Clinical studies in restorative dentistry: new directions and new demands. Dent Mater. 2018 Jan;34(1):1-12. https://doi.org/10.1016/i.dental.2017.08.187

21. Sande FH, Opdam NJ, Rodolpho PA, Correa MB, Demarco FF, Cenci MS. Patient risk factors' influence on survival of posterior composites. J Dent Res. 2013 Jul;92(7 Suppl):78S-83S. https://doi.org/10.1177/0022034513484337

22. Collares K, Correa MB, Bronkhorst EM, Laske M, Huysmans MD, Opdam NJ. A practice based longevity study on single-unit crowns. $J$ Dent. 2018 Jul;74:43-8. https://doi.org/10.1016/i.jdent.2018.05.013 PMID:29800638

23. Deudekom FJ, Postmus I, Ham DJ, Pothof AB, Broekhuizen K, Blauw GJ, et al. External validity of randomized controlled trials in older adults, a systematic review. PLoS One. 2017 Mar;12(3):e0174053. https://doi.org/10.1371/journal.pone.0174053

24. Diemberger I, Massaro G, Reggiani ML, Lorenzetti S, Biffi M, Ziacchi M, et al. Outcomes with dronedarone in atrial fibrillation: what differences between real-world practice and trials? A meta-analysis and meta-regression analysis. Curr Pharm Des. 2017;23(6):944-51. https://doi.org/10.2174/1381612822666161006141512

25. Brooks JM, Chapman CG, Schroeder MC. Understanding treatment effect estimates when treatment effects are heterogeneous for more than one outcome. Appl Health Econ Health Policy. 2018 Jun;16(3):381-93. https://doi.org/10.1007/s40258-018-0380-z

26. Schwartz D, Lellouch J. Explanatory and pragmatic attitudes in therapeutical trials. J Clin Epidemiol. 2009 May;62(5):499-505. https://doi.org/10.1016/i.jclinepi.2009.01.012

27. Tunis SR, Stryer DB, Clancy CM. Practical clinical trials: increasing the value of clinical research for decision making in clinical and health policy. JAMA. 2003 Sep;290(12):1624-32. https://doi.org/10.1001/jama.290.12.1624

28. Blencowe NS, Boddy AP, Harris A, Hanna T, Whiting P, Cook JA, et al. Systematic review of intervention design and delivery in pragmatic and explanatory surgical randomized clinical trials. Br J Surg. 2015 Aug;102(9):1037-47. https://doi.org/10.1002/bjs.9808

29. Patsopoulos NA. A pragmatic view on pragmatic trials. Dialogues Clin Neurosci. 2011;13(2):217-24.

30. Pihlaja J, Näpänkangas R, Raustia A. Early complications and short-term failures of zirconia single crowns and partial fixed dental prostheses. J Prosthet Dent. 2014 Oct;112(4):778-83. https://doi.org/10.1016/i.prosdent.2014.03.008

31. Thorpe KE, Zwarenstein M, Oxman AD, Treweek S, Furberg CD, Altman DG, et al. A pragmatic-explanatory continuum indicator summary (PRECIS): a tool to help trial designers. J Clin Epidemiol. 2009 May;62(5):464-75. https://doi.org/10.1016/i.jclinepi.2008.12.011

32. Loudon K, Treweek S, Sullivan F, Donnan P, Thorpe KE, Zwarenstein M. The PRECIS-2 tool: designing trials that are fit for purpose. BMJ. 2015 May;350 (may08 1):h2147. https://doi.org/10.1136/bmj.h2147

33. Dong Y, Y in W, Hu D, Zhang X, Xu L, Dodds WJ, et al. Remineralization of early caries by chewing sugar-free gum: a clinical study using quantitative light-induced fluorescence. Am J Dent. 2014 Dec;27(6):291-5 
- Practice based research in dentistry: an alternative to deal with clinical questions

34. Laske M, Opdam NJ, Bronkhorst EM, Braspenning JC, Huysmans MC. Risk Factors for Dental Restoration Survival: A Practice-Based Study. J Dent Res. 2019 Apr;98(4):414-22. https://doi.org/10.1177/0022034519827566

35. Mungia R, Funkhouser E, Buchberg Trejo MK, Cohen R, Reyes SC, Cochran DL, et al. Practitioner participation in national dental Practice-Based Research Network (PBRN) Studies: 12-Year Results. J Am Board Fam Med. 2018 Nov-Dec;31 (6):844-56. https://doi.org/10.3122/jabfm.2018.06.180019

36. Makansi N, Bedos C, Allison P. Creating a research network of general dental practitioners: lessons learned from a pilot project. J Can Dent Assoc. 2010;76:a93. 